\title{
TRATAMENTO ORTODÔNTICO PRÉ-PROTÉTICO
}

\author{
PREPROSTHETIC ORTHODONTIC TREATMENT
}

\section{Amanda Pires Vidal', Matheus Silva do Nascimento²}

\section{Resumo}

A Odontologia moderna busca atualmente tratamentos que restaurem função e estética dos elementos dentários, levando também em consideração os fatores biológicos envolvidos na saúde bucal. $\bigcirc$ tratamento reabilitador protético pode ser desafiador, principalmente em pacientes adultos, com perdas dentárias e problemas periodontais. Nesse sentido, a busca por uma somatória de bons resultados leva a equipe a unir especialidades como Periodontia, Dentística, Prótese, Implantodontia, Cirurgia Bucomaxilofacial e Ortodontia. Esta última vem sendo uma grande aliada para a reabilitação protética em etapas de diagnóstico e plano de tratamento, proporcionando melhores prognósticos estéticos e funcionais. $\bigcirc$ presente estudo trata-se de uma revisão da literatura realizada por meio de busca nas bases de dados PubMed e Google Scholar e tem como objetivo abordar os aspectos mais recentes do tratamento ortodôntico pré-protético, enfatizando as principais vantagens, indicações e as formas de estabelecer um plano de tratamento, bem como movimentações ortodônticas em pacientes com agenesia dentária anterior e a utilização de mini-implantes como auxiliares no plano terapêutico. $\bigcirc$ tratamento ortodôntico pré-protético é um tema considerado atual, e a individualidade de cada paciente faz com que a literatura concentre mais artigos de relato de caso, sendo, por isso, necessários mais estudos que englobem protocolos e indicações para os possíveis casos que venham a surgir na prática clínica. Conclui-se que a Ortodontia sendo inserida nas etapas de diagnóstico e plano de tratamento da reabilitação protética traz benefícios de grande valia na busca de um ambiente reabilitador mais fácil e com maiores garantias de sucesso estético e funcional.

Palavras-chave: Ortodontia; Ortodontia corretiva; fechamento de espaço ortodôntico; prótese dentária; planejamento de prótese dentária.

\begin{abstract}
Modern dentistry is currently seeking treatments that restore the function and aesthetics of dental elements, also considering the biological factors involved in oral health. Prosthetic rehabilitation treatment can be challenging, especially in adult patients with tooth loss and periodontal problems. In this sense, the search for a sum of satisfactory results leads the team to integrate specialties such as Periodontics, Dentistry, Prosthodontics, Implantology, Maxillofacial Surgery and Orthodontics. The latter has been a great ally for prosthetic rehabilitation in stages of diagnosis and treatment plan, providing better aesthetic and functional prognoses. The present study is a literature review performed by searching the PubMed and Google Scholar databases and aims to address the most recent aspects of preprosthetic orthodontic treatment, emphasizing the main advantages, indications, and ways of establishing a treatment plan, as well as orthodontic movements in patients with anterior tooth agenesis and the use of mini-implants as an aid in the therapeutic plan. Preprosthetic orthodontic treatment is considered a current topic, and the individuality of each patient means that the literature concentrates more in case report articles, thus further studies are needed that encompass protocols and indications for possible cases that may arise in clinical practice. We conclude that including orthodontics in the stages of diagnosis and treatment plan of prosthetic rehabilitation brings benefits of great value in the search for an easier rehabilitation environment with greater guarantees of aesthetic and functional success.
\end{abstract}

Keywords: Orthodontics; corrective Orthodontics; orthodontic space closure; dental prosthesis; dental prosthesis planning.

I. Especialista em Ortodontia pela Universidade Iguaçu (UNIG), Rio de Janeiro, RJ, Brasil

2. Especialista em Ortodontia pela Universidade Iguaçu (UNIG) e Especialista em Endodontia pela Universidade Federal do Rio de Janeiro (UFRJ).

Clínica de Endodontia - Odontoclínica Central da Marinha (OCM), Rio de Janeiro, Brasil

Como citar este artigo:

Vidal AP, Nascimento MS. Tratamento ortodôntico pré-protético. Rev Nav Odontol. 2021; 48(2): .45-53.

Recebido em: 22/07/2021

Aceito em: 10/09/2021 


\section{INTRODUÇÃO}

A Odontologia moderna preza por tratamentos que restaurem função e estética dos elementos dentários de um paciente, levando também em consideração os fatores biológicos envolvidos na saúde bucal (I). Os tratamentos atualmente buscam interação entre profissionais de forma multidisciplinar, com aplicação de diversas técnicas baseadas em conhecimento científico, permitindo, assim, tratamentos reabilitadores surpreendentes (I-2). Nesse sentido, reuniões de discussão de casos mostram-se muito benéficas no sentido de aprendizado de novas técnicas e ainda de troca de opiniões que vão favorecer o tratamento do paciente (3). Alguns autores destacam, ainda, a importância da Ortodontia nos casos de agenesia de dentes anteriores, que apresentam comprometimento estético importante e muitas vezes tornam o tratamento reabilitador complexo $(4,5)$.

A busca por uma somatória de bons resultados leva a equipe a unir especialidades como Periodontia, Dentística, Prótese, Implantodontia, Cirurgia Bucomaxilofacial e Ortodontia (I-6). Sendo assim, o tratamento reabilitador protético pode ser desafiador em diversos aspectos, principalmente em pacientes adultos, com perdas dentárias e problemas periodontais. Por esse motivo, a Ortodontia cumpre papel fundamental no tratamento e no alcance do objetivo estético final $(3,6,7)$.

$O$ presente trabalho visa, portanto, a abordar uma revisão da literatura, mostrando os aspectos mais recentes do tratamento ortodôntico pré-protético, com uma visão multidisciplinar, enfatizando as principais vantagens, indicações e as formas de estabelecer um plano de tratamento adequado ao paciente de uma forma conjunta e integral, iniciando pelo correto diagnóstico. Além disso, o estudo aborda o papel da Ortodontia em casos de agenesia dentária e a aplicação dos mini-implantes como auxiliares no plano de tratamento.

\section{REVISÃO DA LITERATURA}

Foi realizada uma busca de publicações nas bases de dados PubMed e Google Scholar, utilizando-se as seguintes palavras-chave: preprosthetic; orthodontics; treatment. Foram revisados artigos de língua inglesa sobre o tema, publicados entre 1994 e 2019 (período de I 5 anos). Foi realizada uma seleção inicial de artigos através dos títulos de relevância, seguido da leitura dos resumos. Artigos que tratavam adequadamente do tema da pesquisa foram selecionados para a leitura completa. Foram descartados aqueles artigos contendo casos que não apresentavam reabilitação protética ou tratamento ortodôntico envolvido, totalizando 20 artigos selecionados para compor a revisão de literatura.

A literatura mostra que a Ortodontia tem papel fundamental na reabilitação protética e como é possível unir diversas técnicas para criar espaço, alinhar elementos dentários ou até mesmo contribuir com a relação raiz/ coroa $(2,7)$. No entanto, é importante levar em consideração alguns aspectos antes do planejamento ortodôntico e reabilitador, como a condição periodontal, a idade do paciente, as expectativas envolvidas, o tempo e o custo do tratamento $(3,6)$. Portanto, o correto estabelecimento do diagnóstico seguido de uma correta definição de plano de tratamento podem ser cruciais para alcançar o sucesso do tratamento.

\section{Diagnóstico e Plano de Tratamento}

Estabelecer um diagnóstico preciso é um fator de fundamental importância no estabelecimento do plano de tratamento, principalmente para alcançar o sucesso, unindo fatores como reabilitação biológica, funcional e estética.

Catino et al. estabelecem que o planejamento do tratamento ortodôntico é baseado em uma coleta de dados e exame clínico acurado, acompanhado de exames complementares como radiografias e traçados cefalométricos. Eles sugerem ainda que os parâmetros a serem avaliados devem incluir a funcionalidade de todo o conjunto estomatognático, as condições dos dentes (principalmente a condição da saúde periodontal), o tipo de mordida e de respiração (8).

Alguns autores como Spear et al. propõem um conceito interdisciplinar que preza pela estética em primeiro lugar. Eles alegam que sem considerar os aspectos estéticos o impacto no resultado pode ser catastrófico. Portanto, esse 
grupo de especialistas sugere uma sequência de tratamento que se inicia pela preocupação estética, ou seja, leva em consideração a extensão da exposição da coroa dos dentes anteriores, seja com o lábio em repouso ou durante o sorriso, o posicionamento de linha média (deslocamento para direita ou esquerda), uma correta inclinação dos incisivos e o posicionamento gengival com intuito de trazer um resultado estético satisfatório, antes da preocupação com aspectos biológicos e funcionais (I).

Da mesma forma, ainda em 1997, Almeida et al. já salientavam que dentre os benefícios de lançar mão do tratamento ortodôntico pré-protético estão o reposicionamento dos dentes pilares, a abertura ou fechamento de espaços e a verticalização de dentes inclinados, que facilitam a reabilitação protética, evitando o comprometimento de dentes vitais e sadios, preservando-os de preparos dentários desnecessários e tratamentos endodônticos para garantir a estabilidade das pontes fixas (9).

Normando et al. publicaram um caso tratando especificamente da extrusão ortodôntica no tratamento de invasões de espaços biológicos periodontais. Os autores também definem a cirurgia de aumento de coroa clínica como objetiva e rápida, no entanto, um alongamento da coroa pode gerar um comprometimento estético no que diz respeito à nova inserção gengival. Portanto, quando os casos envolvem região anterior com alta demanda estética, a extrusão ortodôntica pode ser a opção mais vantajosa. Contudo, deve-se levar em consideração a forma externa da raiz do dente a ser extruído, uma vez que uma raiz fina e cônica proporcionará uma região cervical mais estreita após a extrusão dental, gerando preocupação do ponto de vista estético, principalmente referente ao preenchimento do espaço interdental pela papila (10).

A necessidade de tratamento reabilitador pode envolver casos de malformações dentárias (dentes conoides, por exemplo), ausências dentárias, diastemas, contato proximal inadequado e desalinhamento do longo eixo do dente. Em alguns casos, o dentista opta por extrações dentárias seguidas de reabilitação com implantes osseointegrados, porém, essa escolha pode estar equivocada, já que fazer uso de um tratamento ortodôntico pode ser mais simples e rápido (I I).

$\bigcirc$ tratamento ortodôntico compreende, portanto, uma série de abordagens que podem ser aplicadas com objetivos distintos em pacientes com ampla faixa etária. Como já mencionado anteriormente, esse tratamento pode ser benéfico em casos de ausências dentárias por diversas razões, como desnível oclusal, preenchimento de espaços, entre outros. $\bigcirc$ planejamento da etapa da Ortodontia será diretamente influenciado pelo tratamento protético que será realizado posteriormente. De forma sucinta, Catino et al. destacam que o tratamento ortodôntico pré-protético deve ser o mais rápido possível, evitando envolver muitas etapas, e deve focar em um dos objetivos que foram sintetizados na Tabela I (8).

\section{Agenesia dentária anterior e reabilitação}

A agenesia dentária é considerada um dos distúrbios de desenvolvimento mais comuns encontrados na cavidade oral. Dentre as agenesias dentárias, a mais encontrada é a de terceiro molar, seguida dos incisivos laterais (12). Alguns autores relatam também agenesias dos pré-molares como consideravelmente incidentes $(5,13)$.

A ausência de desenvolvimento do elemento dentário permanente é relacionada na literatura com fatores genéticos bem descritos na atualidade $(5, \mid 4)$. Apesar de nem sempre trazer consequências reais ao indivíduo - principalmente quando envolve o terceiro molar — a agenesia dentária do incisivo lateral gera um impacto maior devido ao envolvimento estético, por ser um dente anterior e importante para o alinhamento do sorriso. Essa agenesia pode ser uni ou bilateral, no entanto, quando se apresenta apenas unilateralmente, é comum que o dente contralateral apresente microdontia e seja conoide, uma vez que a base genética envolvida é a mesma (15).

Em termos de tratamento para as agenesias dentárias anteriores, podem-se considerar duas opções: fechamento do espaço com alinhamento e recontorno do dente canino; ou reabilitação protética. Kiliaridis et al. consideraram 12 artigos em sua revisão sistemática, e nesse levantamento de dados os autores mostram que 61,47\% dos casos foram tratados com fechamento do 
Tabela I - Objetivos gerais e indicações das principais movimentações ortodônticas pré-protéticas*

MOVIMENTAÇÃO

Extrusão lenta

\section{OBJETIVO/INDICAÇÕES}

Indicada para tratamento de defeitos em uma ou duas faces dentárias, redução de bolsa periodontal profunda, correção de perfil gengival;

Útil na exposição de lesão de cárie subgengival para tratamento adequado, tratamento de fratura de raiz até determinado ponto, correção de coroa clínica reduzida, tratamento de perfuração radicular. Esses casos devem ser monitorados a cada duas semanas para evitar fibrose gengival;

Utilizada para compensação de perda óssea horizontal, redução de bolsa periodontal, meIhora na inserção do ligamento periodontal, aumento de extensão de coroa clínica, nivelamento de margem incisal, correção de extrusão dentária por falta de antagonista limitada a I-2 mm;

Focada em eliminar interferência funcional, correção de trauma oclusal, corrigir dente de apoio que não estiver paralelo, criar espaço para colocação de implante;

Distribuir as cargas mastigatórias entre os dentes pilares de acordo com o planejamento do tratamento protético posterior.

*Texto adaptado de Catino et al. (8) 
espaço tracionando o canino ortodonticamente e posteriormente fazendo o contorno desse elemento dentário para melhor apresentação estética e funcional (16). De forma interessante, os autores destacam, ainda, que mesmo os casos em que a opção foi a reabilitação protética, 95,5\% deles tiveram uma etapa prévia de tratamento ortodôntico, seja para abertura ou para manutenção de espaço entre o incisivo central e o canino. Esses tratamentos ortodônticos pré-protéticos são muito indicados nesses casos devido à clara tendência do canino mesializar na ausência de contato proximal, buscando, assim, alguma forma de contato com o incisivo central (16).

Ainda considerando a revisão sistemática, o objetivo dos autores foi de comparar os tratamentos de agenesia do incisivo lateral superior com fechamento do espaço com tracionamento ortodôntico do canino ou por reabilitação protética. Eles indicam que a primeira alternativa alcança melhores resultados funcionais, biológicos e estéticos e deve ser a opção de escolha sempre que possível (16).

Por outro lado, algumas considerações devem ser feitas durante a escolha desse tipo de tratamento. Dentre elas, destaca-se a possibilidade de o canino apresentar um volume exagerado e assim exigir um desgaste dentário excessivo $(5,17)$. Outra consideração é quanto à diferença de coloração do dente canino que tende a ser mais amarelado e quanto mais for necessário desgastar o elemento dentário, mais evidente a cor amarelada pode ficar (5). Uma das sugestões dadas por Kiliaridis et al. para contornar esse problema é alterando o torque do canino de modo que reduza a extensão da convexidade labial deste, disfarçando a angulação do dente e requerendo menos desgaste para o recontorno (16). Outras alternativas envolvem o clareamento do dente em questão ou utilização de métodos de restauração estética, como facetas laminadas. Além disso, os autores salientam a importância de verificar a altura da papila interdental e de manter a contenção fixada com resina na palatina dos dentes anteriores por longos períodos (16).

Considerando as possibilidades de tratamento protético após a escolha da intervenção ortodôntica adequada, podem-se considerar três opções:
I. Reabilitação com implante unitário e coroa total;

II. Ponte fixa adesiva;

III. Prótese parcial fixa.

Cada uma das opções tem vantagens e desvantagens, além de considerações a que se deve dar atenção especial no momento da escolha do melhor tratamento ao paciente, como idade, expectativas estéticas e relação custo-benefício, de acordo também com as condições financeiras dele $(16,17)$.

Os implantes unitários aparecem como uma boa opção reabilitadora nos casos de ausência de um elemento dentário. No entanto, é preciso ter cautela no tratamento ortodôntico pré-protético, procurando sempre se certificar radiograficamente, antes de encerrar o tratamento, de que as raízes do canino e do incisivo central estão bem posicionadas para permitir a colocação do implante, principalmente nos casos de abertura de espaço em que o canino apresenta o movimento da coroa mais rápido do que o alinhamento adequado da porção radicular (5). Olsen e Kokich demonstraram que I 1\% dos pacientes em seus estudos apresentaram espaçamento entre raízes inadequado, inviabilizando a colocação do implante unitário ( I 8). Outro fator importante a ser ponderado nessa modalidade de tratamento é a idade do paciente e, por conseguinte, seu completo desenvolvimento ósseo. Nos casos em que esse fator é negligenciado, os implantes podem apresentar infraoclusão (19). Em geral, a escolha pela reabilitação com implantes alcança os requisitos estéticos e funcionais de forma adequada, embora seja uma opção financeiramente mais custosa (16).

A ponte fixa adesiva é definida como uma prótese em que a ausência dentária é substituída por dentes artificiais fixados nos dentes vizinhos, com uso de estrutura metálica ou não (20). $\bigcirc$ uso desse tipo de prótese foi idealizado para solucionar problemas estéticos anteriores e é considerada a opção mais conservadora, pois requer apenas um preparo mínimo dos dentes adjacentes (16). Outras vantagens desse tipo de reabilitação protética consistem no fato de evitar possibilidade de trauma pulpar ou preparo subgengival, o procedimento é mais simples, barato e requer menor tempo do paciente na cadeira odontológica (2l). Apesar dos 
inúmeros benefícios, alguns casos não têm indicação para esse tipo de prótese, como em dentes muito cariados ou restaurados, dentes com alguma mobilidade ou para pacientes com hábitos parafuncionais como o bruxismo $(22,23)$.

A terceira alternativa de reabilitação protética nesses casos é a prótese parcial fixa, que consiste em um tipo de prótese com preparo e cimentação de coroas totais nos dentes adjacentes ao dente ausente. No entanto, para os casos de agenesia de incisivo lateral superior, esse tipo de prótese é o menos recomendado, pois envolve muito desgaste dentário, riscos de envolvimento pulpar, além de ter um custo mais elevado para o paciente (16). Essa reabilitação será mais indicada quando os dentes adjacentes necessitarem de reabilitação mais extensa devido a cáries, fraturas ou pigmentações (16).

Independentemente da escolha do tratamento reabilitador, o tratamento ortodôntico pré-protético desempenha papel fundamental para o planejamento desses casos, pois confere o correto posicionamento dos dentes adjacentes e espaço necessário para a substituição do elemento dentário ausente. Além disso, favorece o bom posicionamento gengival, garantindo os fatores biológicos e estéticos de forma satisfatória.

\section{Uso de mini-implantes no tracio- namento dentário pré-protético}

Com o aumento do número de casos com necessidade de tratamento protético apresentando má oclusão, surgiram novas alternativas no mercado ortodôntico com o intuito de auxiliar o reposicionamento dentário, principalmente em pacientes adultos (24). Existem diversas formas de ancoragem descritas na literatura, como barra lingual e transpalatina, botão de Nance, elásticos intermaxilares e aparelho extrabucal. No entanto, essas opções muitas vezes apresentam desconforto e baixa adesão dos pacientes, além de elevado tempo de tratamento $(25,26)$. Visando a uma modalidade de tratamento mais simples e rápida, começou-se a estudar outras formas de ancoragem intraóssea, surgindo assim os mini-implantes.

Os mini-implantes são descritos como dispositivos de ancoragem temporária, que são fixados no osso alveolar por vestibular e/ou por lingual/palatina. Esses dispositivos apresentam-se na forma de pequenos parafusos compostos de metal biocompatível, como o titânio (25).

Esses dispositivos de ancoragem temporária podem ser instalados por um implantodontista ou até mesmo pelo ortodontista, desde que com treinamento adequado. As principais vantagens dessa modalidade de tratamento envolvem o tamanho reduzido - que traz pouco ou nenhum desconforto ao paciente -, facilidade na instalação, alta resistência às forças ortodônticas, capacidade de receber carga imediata, caso necessário, versatilidade na utilização com diversas possibilidades de aplicações mecânicas ortodônticas, fácil remoção e baixo custo (25). No entanto, o planejamento de colocação dos mini-implantes deve ser feito de forma cautelosa para evitar que estes dispositivos atinjam algum órgão vital, devendo-se realizar exames clínicos e radiográficos que permitam mensurar o espaço entre as raízes, distância de forame, nervos principais e vasos sanguíneos (27).

A aplicação de mini-implantes como dispositivo de ancoragem temporária apresenta inúmeras indicações, que foram detalhadas por Araujo et al. (25). Dentre elas, encontram-se: retração de dentes anteriores (principalmente nos casos de ausência de dentes posteriores que dificulta a ancoragem), mesialização de dentes posteriores, intrusão de incisivos, intrusão de dentes posteriores, correção de plano oclusal (intrusão de elementos dentários pode apresentar uma mecânica complexa que se torna muito facilitada com uso dos mini-implantes), distalização de molares, verticalização e desimpacção de molares, correção de mordida cruzada posterior, e correção de linha média (25).

Soares et al. utilizaram a técnica de verticalização de um molar inferior com mini-implante em um caso complexo e desafiador de um paciente adulto com várias ausências dentárias, quadro de periodontite crônica, má oclusão e abertura de diastemas (28). $\bigcirc$ tratamento interdisciplinar envolveu uma fase inicial de preparo de meio bucal com ajuda da periodontia e cirurgia, eliminando focos de infecção e reestabelecendo a saúde periodontal. Em seguida, o tratamento ortodôntico envolveu quatro eta- 
pas: nivelamento e alinhamento dos elementos dentários; criação de espaços adequados para reabilitação protética; verticalização do terceiro molar inferior com uso de mini-implante ancorado por mola híbrida de aço e NiTi; e criação de guias protrusivas e lateroprotusivas adequadas. Após a finalização do tratamento ortodôntico, foram colocadas próteses implanto-suportadas nas regiões de ausências dentárias e os colos dos dentes anteriores foram preenchidos com resina, a fim de minimizar o comprometimento estético causado pela perda óssea periodontal. Os autores pontuam, por fim, que a interdisciplinaridade e o bom planejamento foram essenciais para o sucesso do tratamento desse caso (28).

Apesar de serem descritas taxas de sucesso entre $80 \%$ e $90 \%$, os mini-implantes podem apresentar falhas em algumas situações ou até mesmo ser contraindicados em determinados pacientes (26). Um dos principais problemas encontrados nas terapias com mini-implantes é a perda de estabilidade do dispositivo, que pode estar sendo ocasionada por diversos fatores, como falha no momento da inserção, localização inadequada, aplicação de força ortodôntica excessiva, ou devido à inflamação dos tecidos peri-implantares, causada por higienização deficiente $(25,26,29)$.

Além disso, outras complicações da terapia envolvem o contato do mini-implante com superfície de raiz dentária, fratura ou perda do parafuso, desenvolvimento de peri-implantite (ou mucosite peri-implantar) e lesões traumáticas em mucosa $(25,29)$. De acordo com o estudo de Chang e Tsengde (2014), os dispositivos de ancoragem temporária são contraindicados em pacientes com problemas de cicatrização ou de defesa imunológica, desordens hematológicas e de coagulação, baixa qualidade óssea e higiene deficiente (26). Ademais, o tratamento pode não ser altamente indicado para crianças e adolescentes em dentição mista e pacientes tabagistas inveterados, cujos índices de inflamação podem ser bastante elevados (26). Diversos autores apontam, ainda, que para alcançar maiores taxas de sucesso, os mini-implantes devem ser posicionados em gengiva inserida, evitando ao máximo a gengiva livre, pois essa região tem maiores chances de inflamação e posterior falha do dispositivo $(25,29)$.

\section{DISCUSSÃO}

A busca pela estética do sorriso e a reabilitação funcional por adultos tem crescido bastante nos últimos anos. Como prova disso, Gorbunkova et al. (2016) mostraram a evolução no número de publicações científicas desde 1965 até momentos mais atuais que abordam as intervenções multidisciplinares, com associação de mais de uma modalidade na busca de alcançar o melhor resultado para o paciente (6). Além disso, outros autores salientam a importância de um plano de tratamento multidisciplinar como pré-requisito para a conclusão satisfatória de tratamentos reabilitadores em pacientes, principalmente adultos, com ausências dentárias e quadros de doença periodontal $(1,19)$.

A literatura não demonstra um protocolo fixo para o tratamento ortodôntico pré-protético, e por isso a definição do plano de tratamento reabilitador protético é de suma importância e impacta diretamente na escolha adequada do tratamento ortodôntico a ser adotado. Entretanto, Spear et al. determinam que a estética deve ser considerada em primeiro lugar, em detrimento dos aspectos biológicos e funcionais ( I). Muito embora esse posicionamento possa gerar certa estranheza, esses últimos fatores não são negligenciados, sendo salientado pelos autores igualmente sua importância (I).

$\bigcirc$ tratamento ortodôntico pré-protético apresenta vários benefícios descritos por diversos autores. Dentre eles pode-se destacar a preservação da vitalidade de alguns dentes por reduzir a necessidade de desgastes para cimentação de próteses fixas, recuperação periodontal e de inserção gengival, realinhamento de plano oclusal, redução da necessidade de intervenções cirúrgicas, como nos casos de aumento de coroa clínica ou extrações dentárias ( I, 7, $9,28)$. Sendo assim, muitas vezes o tratamento ortodôntico mostra-se uma alternativa mais eficaz, barata e conservadora ( I I).

Uma das reabilitações em que mais se discute o papel do tratamento ortodôntico prévio é no caso de agenesia do incisivo lateral superior. Diversos autores discutem as formas de reabilitação e, na maioria delas, o benefício maior é encontrado quando o canino é tracionado, sendo reposicionado para o lugar do incisivo lateral, sofrendo posteriormente um recontorno 
adequado $(5,16)$. Kiliaridis et al. $(20 \mid 6)$ ponderam, em sua revisão, que um paciente que é submetido a fechamento de espaço por Ortodontia tem uma melhor saúde periodontal, apresentando menores índices de biofilme e sangramento gengival. Uma alternativa a se considerar nesses casos também é a manutenção ou, se necessário, a abertura de espaço entre o incisivo central e o canino para posterior colocação de implante unitário (16). No entanto, a reabilitação com implantes deve ser muito bem estudada e apenas realizada no momento certo, ou seja, após finalização do desenvolvimento ósseo, para evitar casos de infraoclusão do implante (19).

Segundo estudos recentes apontam, uma das melhores opções reabilitadoras ainda é a ponte fixa adesiva, pois é uma alternativa que apresenta menos desgaste dentário, pouco invasiva, de custo reduzido, além de mostrar resultados estéticos bastante satisfatórios (I6,2I). Como alguns casos possuem contraindicações para esse tipo de tratamento reabilitador (22, 23), outra possibilidade é a prótese parcial fixa, que pode alcançar resultados estéticos satisfatórios, principalmente em pacientes cujos dentes anteriores apresentem cáries ou fraturas extensas ( I 6). Apesar de a escolha do tratamento reabilitador poder variar, o tratamento ortodôntico pré-protético é fundamental e garante o correto posicionamento dos dentes adjacentes ou espaço necessário para a substituição do incisivo lateral ausente. Dentre outros benefícios, esse planejamento e tratamento prévio favorecem fatores biológicos e estéticos importantes para alcançar o resultado ideal (3).

Com o aumento da demanda de pacientes adultos apresentando exigência de movimentos muito difíceis no campo da Ortodontia, surgiram os mini-implantes como agentes facilitadores dos movimentos (25). Os autores concordam que esses dispositivos de ancoragem temporária auxiliam de forma importante na movimentação dentária de molares que, pela localização ao final da arcada, não possibilitam ancoragem posterior adequada pelos métodos tradicionais para movimentos como intrusão e verticalização do elemento dentário $(24,28)$. A literatura destaca que apesar de exigir cautela no momento da instalação devido ao risco de atingir estruturas vitais, a técnica que utiliza o mini-implante apresenta altas taxas de sucesso e tem sido bastante recomendada na prática clínica $(25-27,30)$.

O tratamento ortodôntico pré-protético já se mostra uma alternativa de suma relevância, principalmente pelo aumento da procura de reabilitações protéticas complexas por pacientes adultos. Em vista disso, o presente estudo salienta sua relevância na abordagem de tópicos que podem contribuir com a literatura na elucidação de alternativas de tratamento. Apesar de ser um tema considerado atual e pertinente, a individualidade de cada paciente faz com que a literatura concentre mais artigos de relato de caso, sendo necessários mais estudos que concentrem protocolos e indicações para os possíveis casos que venham a surgir na prática clínica, auxiliando o especialista na escolha do melhor tratamento para o paciente.

\section{CONCLUSÃO}

Este trabalho concluiu, portanto, que a Ortodontia sendo inserida nas etapas de diagnóstico e plano de tratamento da reabilitação protética traz benefícios de grande valia na busca de um ambiente reabilitador mais fácil e com maiores garantias de sucesso estético e funcional, especialmente em casos mais complexos de ausências e agenesias dentárias. $\bigcirc$ uso de mini-implantes se mostra uma alternativa atual para movimentos mais pontuais de forma mais rápida e efetiva.

Os autores declaram não haver conflito de interesse.

\section{Autora de correspondência:}

Amanda Pires Vidal

Rua Alfredo Menezes, 1205 - Bacaxá, Saquarema - RJ

CEP 28994I86

vidal.amanda@gmail.com

\section{REFERÊNCIAS BIBLIOGRÁFICAS}

I. Spear FM, Kokich VG, Mathews DP. Interdisciplinary management of anterior dental esthetics. J Am Dent Assoc. 2006; 137(2): 160-169.

2. Silva-Coll J, Hernández-Orsini R,Wang CW. Corticotomy-assisted adult rapid maxillary arch expansion and ridge augmentation: An interdisciplinary case report with 7-year follow-up. Am J Orthod Dentofacial Orthop. 20 I9; I 56(2):266-274. 
3. Spear FM. My growing involvement in dental study groups. J Am Coll Dent. 2002; 69(4):22-4.

4. Kokich VG. Maxillary Lateral Incisor Implants: Planning With the Aid of Orthodontics.J Oral Maxillofac Surg. 2004; 62:48-56, Suppl 2.

5. Krassnig M, Fickl S. Congenitally missing lateral incisors--a comparison between restorative, implant, and orthodontic approaches. Dent Clin North Am. 20 I I; 55(2):283-99.

6. Gorbunkova A, Pagni G, Brizhak A, Farronato G, Rasperini G. Impact of Orthodontic Treatment on Periodontal Tissues: A Narrative Review of Multidisciplinary Literature. Int J Dent. 20 I6; 2016: 4723589.

7. Konstantinova D, Arnautska $H$. Orthodontic-prosthetic approach in the treatment of complex clinical cases. J of IMAB. 20। 4; 20(I):469-472.

8. Catino B, Pomatto E,Preti S, Salvi S. Preprosthetic Orthodontics and segmental osteotomy. In: PRETI, Giulio. Prosthetic Reabilitation. Bologna: Quintessence. 2008; p. I27- 148.

9. Almeida RR, Bonfante G, Neto GI,Almeida MR.A Inter-relação Ortodontia e Prótese: apresentação de um Caso Clínico. Rev Dental Press De Ortod E Ortop Maxil. 1997; 2(4): I3- 19.

10. Normando ADC, Simone JL, Soares MS, Tortamano N. A extrusão ortodôntica como recurso no tratamento das invasões dos espaços biológicos periodontais: indicação clínica e divulgação de um método simplificado de tratamento. J Bras Ortodon Ortop Facial. 2004; 9(53): 502-5 I 0.

11. Freitas-Junior AC, Silva AM. Pre-Prosthetic Orthodontic Treatment Using Personalized Elastic Separators for Optimization of Emergence Profile in Esthetic Crowns: A Clinical Report. J Prosthodont. 20 12; 2 I ( I):52-5.

12. Vastardis $H$. The genetics of human tooth agenesis: new discoveries for understanding dental anomalies. Am J Orthod Dentofacial Orthop. 2000; 1 1 7(6):650-6.

13. Muller TP, Hill IN, Peterson AC, Blayney JR. A survey of congenitally missing permanent teeth. J Am Dent Assoc. 1970; $81(1): 101-7$.

14. Alves-Ferreira M, Pinho T, Sousa A, Sequeiros J, Lemos C, Alonso I. Identification of genetic risk factors for maxillary lateral incisor agenesis. J Dent Res. 20 I4; 93(5):452-8.

15. Svinhufvud E, Myllärniemi S, Norio R. Dominant inheritance of tooth malpositions and their association to hypodontia. Clin Genet. 1988; 34(6):373-8I.

16. Kiliaridis S, Sidira M, Kirmanidou Y, Michalakis K. Treatment options for congenitally missing lateral incisors. Eur J Oral
Implantol. 20 I6; Suppl I:S5-24.

17. Jatol-Tekade S, Tekade SA, Khetrapal S, Sarode SC, Patil S. Multidisciplinary approach to bilaterally missing lateral incisors: A case report. ClinPract. 20 19; 9(1): 1086.

18. Olsen TM, Kokich VG. Postorthodontic root approximation after opening space for maxillary lateral incisor implants. Am J Orthod Dentofacial Orthop. 20 10; 137(2): I58-9.

19. Bernard JP, Schatz JP, Christou P, Belser U, Kiliaridis S. Longterm vertical changes of the anterior maxillary teeth adjacent to single implants in young and mature adults. A retrospective study.J Clin Periodontol. 2004; 31 ( I I): 1024-8.

20. Jorge JH, Neppelenbroek KH, Campanha NH, Segalla JCM, Giampaolo ET. Considerações gerais sobre prótese fixa adesiva. Arq Odontol. 201 I; 47(3): 170- 177.

21. Rosentiel S, Land M, Fujimoto J. Contemporary Fixed Prosthodontics. St Louis: Elsevier, p. 8| 2. 2006.

22. Kinzer GA, Kolkich VO. Managing congenitally missing lateral incisors. Part II: tooth support restorations. J Esthet Rest Dent. 2005; 17: 76-84.

23. El-Mowafy O, Rubo MH. Resin-bonded fixed partial dentures: a literature review with presentation of a novel approach. Int J Prosthodont. 2000; I 3: 460-467.

24. Leite GN.Viabilização protética com o auxllio da ortodontia. 2009. 29 pág. (Curso de Especialização em Prótese Dentária) - Universidade Federal de Santa Catarina, Florianópolis, 2009.

25. Araujo TM, Nascimento MHA, Bezerra F, Sobral MC. Ancoragem esquelética em Ortodontia com miniimplantes. R Dental Press Ortodon Ortop Facial. 2006; I I (4): I26-I56.

26. Chang HP, Tseng YC. Miniscrew implant applications in contemporary orthodontics. Kaohsiung J of Med Sci. 2014; 30(3): I I I- I I5.

27. Liou EJW, Pai BCJ, Lin JCY. Do miniscrews remain stationary under orthodontic forces? Am J Orthod Dentofacial Orthop. 2004; I 26(I):42-7.

28. Soares MS, Morea C, Romano MM, Adde CA, Dominguez GC. Abordagem interdisciplinar em reabilitação bucal. Rev Assoc Paul Cir Dent. 20I2; 66(4):260-7.

29. Mizrahi E, Mizrahi B. Mini-screw implants (temporary Anchorage devices): orthodontic and pre-prosthetic applications. J Orthod. 2007; 34(2): 80-94.

30. Poggio PM, Incorvati C, Velo S, Carano A. "Safe Zones": A guide for miniscrew positioning in the maxillary and mandibular arch. Angle Orthod. 2006; 76:191-197. 